\title{
UN MARCO TEÓRICO PARA ANALIZAR EL SESGO DE GÉNERO EN LA INVESTIGACIÓN EPIDEMIOLÓGICA: HACIENDO VISIBLE LO INVISIBLE
}

\author{
ELISA CHILET ROSELL \\ Universidad de Alicante
}

Recibido/15/07/2011

Aceptado/25/09/2011

\section{Referencia del artículo comentado}

RUIZ CANTERO, María Teresa, VIVES CASES, Carmen, ARTACOZ Lucia, DELGADO Ana, GARCIA CALVENTE María del Mar, MIQUEO Consuelo, MONTERO Isabel, ORTIZ MONCADAS Rocio, RONDA PÉREZ Elena, RUIZ PÉREZ Isabel y VALLS Carme. «A framework to analyse gender bias in epidemiological research» Revista Journal of Epidemiology and community health, 2007: 61 (supl. II) pp. ii46-ii53.

\section{Resumen}

El diseño y el análisis de las investigaciones pueden causar errores sistemáticos producidos por la insensibilidad de género y el androcentrismo. Los sesgos de género en investigación pueden ser definidos como una aproximación sistemáticamente errónea dependiente de la perspectiva de género que se aplica por el constructo social en el que se desarrollan las investigaciones, la cual mira a las mujeres y a los hombres en unas ocasiones como similares cuando no lo son, y en otras como diferentes, y también es incorrecto.

Los sesgos de género se pueden desarrollar en el contexto de descubrimiento (desarrollo de la hipótesis de trabajo), pero también se pueden producir en el contexto de la justificación (en la metodología). De hecho, uno de los principales efectos de los sesgos de género en investigación es un conocimiento parcial o incorrecto de los resultados, los cuales son sistemáticamente diferentes de los valores reales. 
Este artículo discute algunas formas de sesgos conceptuales y metodológicos que pueden afectar la salud de las mujeres. Se propone un marco de análisis de los sesgos de género en el diseño y en el análisis de las investigaciones llevadas a cabo en problemas de salud que pueden ser padecidos por hombres y mujeres, y en problemas de salud específicos de las mujeres.

Mediante ejemplos, este marco de trabajo tiene el objetivo de mostrar las diferentes perspectivas teóricas en un contexto de investigación clínico y en el social, en que los sesgos de selección, medición y confusión se producen como resultados de la insensibilidad de género. Finalmente, este artículo subraya la importancia de reexaminar los resultados con el fin de ser reinterpretados para producir nuevo conocimiento basado en la perspectiva de género. 


\section{Introducción}

A pesar de que tradicionalmente se ha considerado la ciencia como objetiva, la epistemología feminista ha considerado que muchas de sus aplicaciones, formas de definir los problemas de investigación y de diseñar experimentos, modos de construir y conferir significados a la investigación se han desarrollado de forma sesgada a favor del género masculino ${ }^{1}$.

Ante esta afirmación una principiante en salud pública, puede preguntarse cómo identificar el sesgo de género en la investigación pero también, cómo no cometer este error en futuras investigaciones. Las autoras del artículo ${ }^{2}$ propuesto para este ensayo presentan un marco de gran utilidad para el análisis del sesgo de género en investigación, que se traducirá en errores en la atención sanitaria. Además de recoger evidencia científica sobre el sesgo de género, abre el camino a la reflexión, y sin duda, abre la puerta a futuras investigaciones.

\section{Desarrollo}

El sesgo de género en investigación se define como «un error sistemático en el enfoque de género relacionado con el constructo social, que erróneamente considera a hombres y mujeres iguales/diferentes tanto en la exposición a factores de riesgo como en la historia natural y social de la enfermedad (inicio o pródromos, fase consolidada, respuesta a los tratamientos y pronóstico» ${ }^{3}$. En la tabla 1, se muestra de manera esquemática el marco propuesto por este artículo en el que la insensibilidad de género produce sesgos epidemiológicos en investigación, tanto en contextos clínicos como sociales, lo cual puede influir en los resultados, de manera positiva, negativa o neutral.

1. HARDING, Sandra. The science question in feminism. New York: Cornell University Press, Ithaca, 1993.

2. Ruiz Cantero, Maria Teresa; Vives CASES, Carmen; ArTazcoz, Lucia et al. A Framework to analyse gender bias in epidemiological research. J Epidemiol Community Health 61(suppl II) (2007) pp. ii46-ii53.

3. Ibidem. 


\begin{tabular}{|c|c|c|c|c|c|c|c|c|c|}
\hline 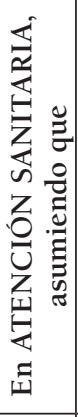 & 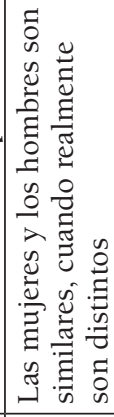 & & & & 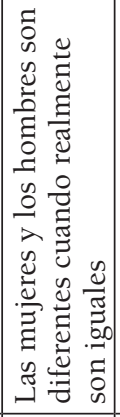 & & & & 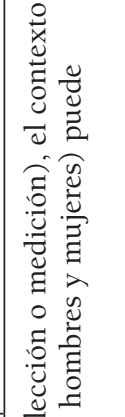 \\
\hline 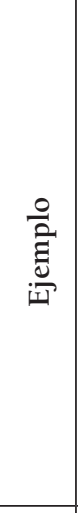 & 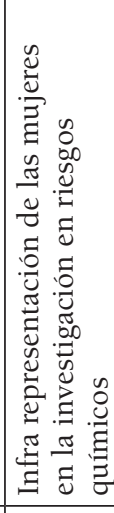 & 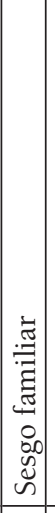 & 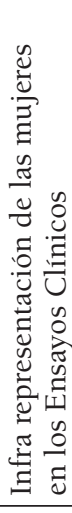 & 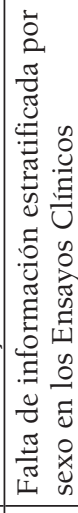 & 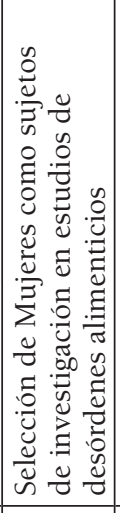 & 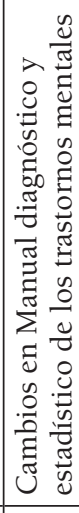 & 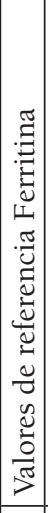 & 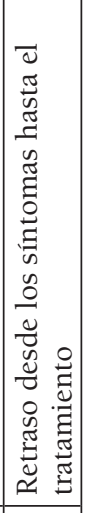 & 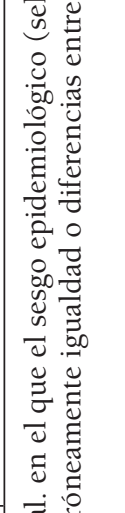 \\
\hline 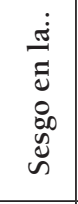 & 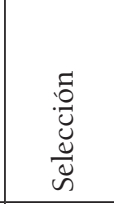 & 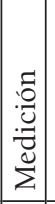 & 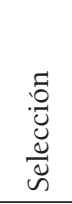 & 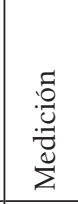 & 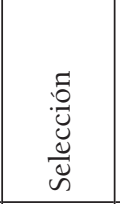 & 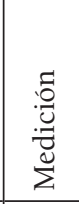 & 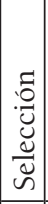 & 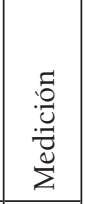 & 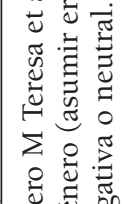 \\
\hline 苞 & $\begin{array}{l}\vec{\pi} \\
\text { O } \\
\stackrel{n}{ }\end{array}$ & & 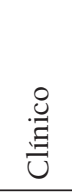 & & 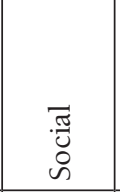 & & 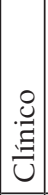 & & 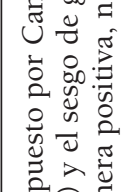 \\
\hline 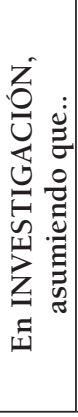 & 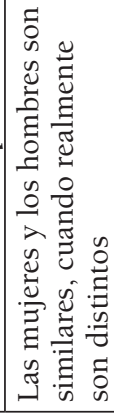 & & & & 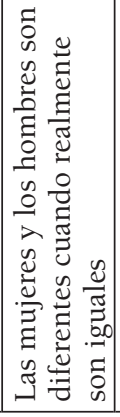 & & & & 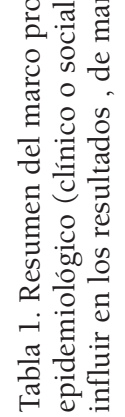 \\
\hline
\end{tabular}

Feminismo/s 18, diciembre 2011, pp. 353-363 
¿Cuál es la importancia de un marco para detectar el sesgo de género en investigación?

Desde la publicación en 1990 del informe de la Oficina Regional de la Organización de Mundial de la Salud ${ }^{4}$, se debate en las revistas científicas sobre el sesgo de género en la atención sanitaria, alegando que la evidencia en la que se sustenta la medicina puede ser incompleta debido a que las herramientas de investigación fallan sistemáticamente en incluir las diferencias por sexo en el diseño del estudio y el análisis de los resultados.

La relación entre el sexo, el género y la salud es compleja. El hecho de contar con un marco teórico nos permite hacer visibles los sesgos de género presentes en la investigación, así como sus consecuencias en la atención sanitaria y en la salud. Hacer visible la presencia de estos sesgos en el diseño, análisis e interpretación de las investigaciones, nos permitirá ocupar los vacíos de conocimiento sobre la salud de las mujeres, y comprender las necesidades de salud de estas; así como sus riesgos. No se trata de simplemente señalar los errores o corregir lo que históricamente se ha hecho de manera sesgada, se trata de mejorar la atención sanitaria y las intervenciones.

Como propusieron M Teresa Ruiz Cantero y Lois M Verbrugge (1997) el marco teórico expuesto en este artículo reflexiona sobre cómo los sesgos epidemiológicos pueden darse por dos vías: considerando que los hombres y las mujeres son iguales cuando realmente son diferentes o considerando que los hombres y las mujeres son diferentes cuando realmente son iguales. El contexto en el que se puede producir el sesgo de género es más amplio que el propuesto por el marco reduccionista biomédico (que no incluye la influencia social), puede ser en la investigación clínica o en la investigación social. El sesgo de género puede producirse en la medición o en la selección de los sujetos de investigación.

Quizás el ejemplo más conocido sobre falta de conocimiento de una enfermedad en mujeres sean las enfermedades cardiovasculares. Tradicionalmente se ha considerado una enfermedad propia de los hombres, obviando que en las mujeres es la primera causa de muerte $e^{6}$. El articulo del cual propongo su lectura y análisis, propone otros ejemplos de sesgo de género en investiga-

4. WhiteHEAD, Margaret. The concepts and principles of equity and health. Copenhagen: WHO Regional Office for Europe; 1990. (Disponible en http://salud.ciee.flacso.org.ar/ flacso/optativas/equity_and_health.pdf, consultado el 7/7/2011)

5. RUIZ, M Teresa; VerbrugGe, Lois M. A two way view of gender bias in medicine. J Epidemiol Community Health. 51 (1997) pp. 106-9.

6. Lloyd-JONES, D; AdAMS, Robert; CARNETHON, Mercedes et al. Heart disease and stroke statistics: 2009 update: a report from the American Heart Association Statistics Committee and Stroke Statistics Subcommitte. Circulation 119 (2009) pp. e21-e181.

Feminismo/s 18, diciembre 2011, pp. 353-363 
ción, quizás menos conocidos, pero no por ellos menos importantes. A través de estos ejemplos, el o la lectora del artículo podrá seguir la argumentación del marco teórico y metodológico expuesto.

\subsection{Asumir erróneamente que las mujeres y los hombres son similares}

Por ejemplo, el artículo nos presenta el denominado «sesgo familiar» como muestra del sesgo de medición en epidemiología social. En este ejemplo se expone como la insensibilidad de género lleva a considerar como unidad de investigación la familia al estudiar los cuidados informales, haciendo invisible el hecho de que son principalmente las mujeres quienes se dedican al cuidado de los menores y de las personas mayores?

Como hemos expuesto anteriormente, la importancia de identificar y hacer visible estos sesgos es mejorar el conocimiento sobre las necesidades de salud de las mujeres; así como de los hombres. En este sentido, cabe destacar el esfuerzo realizado en los cuestionarios de la Encuesta Nacional de Salud (ENS). El análisis de la ENS-2003 evidenció que no era posible determinar por completo indicadores de desigualdad social entre ambos sexos, como son la situación de las amas de casa y de doble jornada ${ }^{8}$. A diferencia de las ENS previas, en la de 2006 se pregunta por la identidad de la persona cuidadora principal de menores de 15 años, mayores de 74 y personas con discapacidad, así como con quién comparte estas tareas".

En cuanto al sesgo de selección en epidemiología social, se expone la infra representación de mujeres en investigación sobre los riesgos químicos. Se consideran los mismos valores para hombres y mujeres en los valores umbral de exposición en las evaluaciones de riesgos químicos en salud laboral, debido a la falta de información específica en mujeres, a pesar de que son conocidas las diferencias por sexo en el metabolismo. Así mismo, no se consideran características relevantes de profesiones llevadas a cabo mayoritariamente por mujeres, como son las peluqueras con más horas de exposición y exposición

7. KotKamp-Mothes, N., Slawinsky, D., Hindermann, S., Strauss, B. Coping and psychological well being in families of elderly cancer patients. Crit Rev Oncol Hematol 55 (2005) pp. 213-29.

8. Ruiz Cantero, M. Teresa, Papí Gálvez, Natalia, Carbrera Ruiz, Virginia, Ruiz MartíNEZ, Ana, Álvarez-DARDET DíAz, Carlos. Los sistemas de género y/en la Encuesta Nacional de Salud. Gac Sanit 20 (2006) pp. 427-34.

9. Ruiz-Cantero, M. Teresa, Carrasco-Portiño, Mercedes, Artazcoz, Lucía. Logros y retos de género de la Encuesta Nacional de Salud de 2006: análisis de los cuestionarios de adultos y hogar. Gac Sanit. 25 (2010): 6-12. 
cutánea (no sólo inhalación) ${ }^{10}$. El hecho de hacer visible este sesgo de género ha propiciado que en los últimos años haya aumentado la investigación sobre los riesgos asociados a esta profesión ${ }^{11},{ }^{12},{ }^{13},{ }^{14}$.

La participación de mujeres en Ensayos Clínicos, sirve de ejemplo para dos tipos de sesgo. En primer lugar el sesgo de selección en epidemiología clínica. Durante años, las mujeres fueron excluidas de los ensayos clínicos por temor a los efectos que la exposición a nuevos fármacos pudiese tener en el embarazo. No fue hasta los años 90, tras el trabajo reivindicativo de grupos feministas, que National Health Institutes ${ }^{15}$ y la Food and Drug Administration ${ }^{16}$ reformularon sus guías para que las mujeres participaran en los ensayos clínicos. Aunque la participación de las mujeres ha aumentado, su participación en ensayos de fase I y fase $\mathrm{II}^{17},{ }^{18}$ es menor, por tanto, información referente a seguridad puede ser desconocida.

Cabe llamar la atención sobre los ensayos clínicos revisados para la elaboración de las Guías publicadas por la American Heart Association para la prevención de enfermedad cardiovascular en mujeres ${ }^{19}$. Aunque supone un gran avance el hecho que la American Heart Association publique unas guías específicas para mujeres, en los ensayos analizados para su elaboración, la participación de las mujeres aumentó desde el 18\% en 1970 a un 34\% en 2006. Es

10. Van Der Wal, Jan F; Hoogeveen, Ank W.; Moons, André M.; Wouda, Paul. Investigation on the exposure of hairdressers to chemical agents. Environment International 23 (1997) pp. 433-9.

11. Ronda, Elena, García, Ana M., SÁnchez-Paya, Jose, Moen, Bente E. Menstrual disorders and subfertility in Spanish hairdressers. Eur J Obstet Gynecol Reprod Biol 147 (2009) pp. 61-4.

12. Takkouche, Bahi, Regueira-Méndez, Carlos, Montes-Martínez, Agustín N. Risk of cancer among hairdressers and related workers: a meta-analysis. Int J Epidemiol 38 (2009) pp. 1512-31.

13. Peretz, Jackye; Gallicchio, Lisa; Miller, Susan et al. Infertility among cosmetologists. Reproductive Toxicology 28 (2009) pp. 359-64.

14. MA, C-M, LIN, L-Y, CHEN, H-W et al. Volatile organic compounds exposure and cardiovascular effects in hair salons. Occup Med. 60(2010) pp. 624-30.

15. NIH Revitalization Act, Subtitle B, Part 1, Section 131-133, June 10, 1993.

16. FOOD AND DRUG ADMINISTRATION Guideline for the study and evaluation of gender differences in the clinical evaluation of drugs. Fed Reg 1993;58:39409-11

17. Chilet-Rosell, Elisa; Ruiz-Cantero, M. Teresa; HORGA, Jose Francisco. Women's health and gender-based clinical trials on etoricoxib: methodological gender bias. $J$ Public Health 31 (2010) pp. 434-445.

18. FleisCH, Jeanette; FleisCh, Markus; Thürmann, Petra A. Women in early-phase clinical drug trials. Have things changed over the past 20 years? Clin Pharmacol Ther 78 (2005) pp. 445-52.

19. Mosca, Lori; ApPel, Lawrence J.; Benjamin, Emelia J. Evidence-based guidelines for cardiovascular disease prevention in women. J Am Coll Cardiol 43 (2004) pp. 900-921.

Feminismo/s 18, diciembre 2011, pp. 353-363 
decir, aunque la participación de mujeres en ensayos clínicos ha aumentado, no representan la población que padecerá enfermedades cardiovasculares.

La falta de información estratificada por sexo es un claro ejemplo de sesgo de medición en epidemiología clínica, Aunque las mujeres están siendo incluidas en los ensayos clínicos, los resultados por sexo a penas se incluyen en los ensayos clínicos publicados. En este sentido volvemos a llamar la atención sobre los ensayos clínicos analizados en las Guías publicadas por la American Heart Association, en las que los sólo el 31\% de los ensayos clínicos analizados incluía el análisis de los resultados por sexo.

\subsection{Asumir erróneamente que las mujeres y los hombres son diferentes}

No siempre el sesgo de género perjudica a las mujeres ${ }^{20}$. En el caso expuesto como ejemplo de sesgo de selección en epidemiología social, las investigaciones sobre los desórdenes alimenticios suelen considerar que sólo afecta a mujeres, pudiendo provocar una infraestimación de la prevalencia en hombres.

El sesgo de medición en epidemiología social, se muestra en el ejemplo del análisis del manual Diagnostic and statistical manual of mental disorders $(D S M)^{21}$. Tradicionalmente se considera que el estrés postraumático es más en común en mujeres debido a su mayor vulnerabilidad biológica, diferentes estilos para sobrellevar el estrés o historia familiar ${ }^{22}$. Para esta afirmación, no se ha tenido en cuenta la importancia de la definición de evento traumático. En su última edición (DSM-IV), se incluyen nuevos tipos de eventos traumáticos más comunes en mujeres (como la violación o abuso sexual), asumiendo que la afirmación sobre la menor exposición a eventos traumáticos en mujeres se debe a un sesgo en el instrumento de medida (en los cuestionarios) que no incluían riesgos relevantes para las mujeres.

En epidemiología clínica, como sesgo de selección se presenta el caso de la ferritina en sangre, que presenta como normal valores bajos en mujeres, asumiendo que estos valores son normales en mujeres debido a la pérdida de sangre que se produce durante la menstruación y obviando que puede afectar

20. Doyal, Lesley. Sex, gender, and health: the need for a new approach. BMJ 323(2001) pp. 1061.

21. AMERICAN PSYCHIATRIC ASSOCIATION. Diagnostic and statistical manual of mental disorders. Washington: APA, 1994.

22. BResLau, N.; DAVIS, G. C.; ANDRESKi P. et al. Sex differences in post-traumatic stress disorder. Arch Gen Psychiatry 54 (1997) pp. 1044-8. 
a su calidad de vida ${ }^{23}$. Como sesgo de selección, se presenta el retraso entre la presencia de síntomas y la obtención de tratamiento; en concreto, en enfermedades cardiovasculares. El estudio IBERICA (que incluye 8200 infartos de miocardio en España) muestra que las mujeres experimentan mayores retrasos desde la presentación de síntomas hasta el ingreso hospitalario. Sus consecuencias son importantes, como se ha observado en la evitable mayor tasa de letalidad hospitalaria en mujeres que en hombres por infarto de miocardio ${ }^{24}$.

\section{Conclusiones}

«La ceguera o el sesgo de género constituye un grave defecto que introduce una forma muy seria de error dentro de la investigación científica» ${ }^{25}$. Retomando la argumentación del Empirismo Feminista, que afirma que el sesgo de género se produce al no aplicar las normas científicas de modo correcto, el hacer visibles estos errores sistemáticos en la investigación y sus consecuencias, se resalta la necesidad de eliminar el sesgo de género en el desarrollo de hipótesis e interpretación de resultados.

En este sentido, me gustaría acabar este ensayo con un toque de optimismo. Como se señalaba al comienzo de este ensayo, se trata de producir conocimiento que cubra los vacíos existentes y mejorar la atención sanitaria haciendo que ésta sea basada en la mejor evidencia posible. Por ello, quisiera destacar las acciones llevadas a cabo en la legislación española en los últimos años. En primer lugar la Ley Orgánica 3/200, de 22 de marzo, para la igualdad efectiva de mujeres y hombres, que afirma que siempre que sea posible la desagregación por sexo de los datos contenidos en registros, encuestas, estadísticas u otros sistemas de información médica para permitir el análisis de género. En la misma línea, se aprobó la Ley 14/2011, de 1 de junio, de la Ciencia, la Tecnología y la Innovación que instaura la perspectiva de género como una categoría transversal en la investigación científica y técnica, que debe ser tenida en cuenta en todos los aspectos del proceso para garantizar la igualdad efectiva entre hombres y mujeres.

23. Fleming, D. J.; Jacques, P. F; Dallal, G. E.; Tucker, K. L. \& Wilson, P. W. Dietary determinants of iron stores in a free-living elderly population: The Framingham Heart Study. Am J Clin Nutr, 67(1998) pp. 722-733.

24. Segura, Andreu, ZuRRiaga, O, ROHLFS, Isabel et al. diferencias por género en las características, manejo y letalidad-complicaciones en pacientes con infarto agudo de miocardio (IAM): Estudiolberica. Gac Sanit 16 (Suppl 1) (2002) pp. 90.

25. EICHLER, Magrit; ReISMAn, Anna Lisa; BorInS, Elaine. Gender Bias in Medical Research. Women and Therapy 12 (1992) pp. 61-70.

Feminismo/s 18, diciembre 2011, pp. 353-363 


\section{Referencias bibliográficas}

AMERICAN PSYCHIATRIC ASSOCIATION. Diagnostic and statistical manual of mental disorders. Washington: APA, 1994.

Breslau, N.; DAVIS, G. C.; ANDRESKi P. et al. Sex differences in post-traumatic stress disorder. Arch Gen Psychiatry 54 (1997) pp. 1044-8.

Chilet-Rosell, Elisa; RuIz-CAnTero, M. Teresa; HorGA, Jose Francisco. Women's health and gender-based clinical trials on etoricoxib: methodological gender bias. J Public Health 31 (2010) pp. 434445.

DOyAL, Lesley. Sex, gender, and health: the need for a new approach. BMJ 323(2001) pp 1061

EICHler, Magrit; ReISMAn, Anna Lisa; Borins, Elaine. Gender Bias in Medical Research. Women and Therapy 12 (1992) pp 61-70.

FleisCH, Jeanette; FleisCh, Markus; Thürmann, Petra A. Women in early-phase clinical drug trials. Have things changed over the past 20 years? Clin Pharmacol Ther 78 (2005) pp:445-52.

Fleming, D. J.; Jacques, P. F; Dallal, G. E.; Tucker, K. L. \& Wilson, P. W. Dietary determinants of iron stores in a free-living elderly population: The Framingham Heart Study. Am J Clin Nutr, 67(1998) pp 722-733.

FOOD AND DRUG ADMINISTRATION Guideline for the study and evaluation of gender differences in the clinical evaluation of drugs. Fed Reg 1993;58:39409-11

HARDING, Sandra. The science question in feminism. New York: Cornell University Press, Ithaca, 1993

Kotkamp-Mothes, N., Slawinsky, D., Hindermann, S., Strauss, B. Coping and psychological well being in families of elderly cancer patients. Crit Rev Oncol Hematol 55 (2005) pp 213-29.

Lloyd-Jones, D; AdAms, Robert; CARnethon, Mercedes et al. Heart disease and stroke statistics: 2009 update: a report from the American Heart Association Statistics Committee and Stroke Statistics Subcommitte. Circulation 119 (2009) pp.e21-e181.

MA, C.-M., LiN, L.-Y., CHEN, H.-W. et al. Volatile organic compounds exposure and cardiovascular effects in hair salons. Occup Med. 60 (2010) pp 624-30.

NIH Revitalization Act, Subtitle B, Part 1, Section 131-133, June 10, 1993.

PereTZ, Jackye; GALLiCCHIO, Lisa; MiLler, Susan et al. Infertility among cosmetologists. Reproductive Toxicology 28 (2009) pp 359-64.

Ronda, Elena, García, Ana M., SÁnchez-Paya, Jose, Moen, Bente E. Menstrual disorders and subfertility in Spanish hairdressers. Eur J Obstet Gynecol Reprod Biol 147 (2009) pp 61-4. 
Ruiz-Cantero, M. Teresa, Carrasco-Portiño, Mercedes, Artazcoz, Lucía. Logros y retos de género de la Encuesta Nacional de Salud de 2006: análisis de los cuestionarios de adultos y hogar. Gac Sanit. 25 (2010): 6-12.

Ruiz Cantero, Maria Teresa; Vives Cases, Carmen; ArTazCoz, Lucia et al. A Framework to analyse gender bias in epidemiological research. J Epidemiol Community Health 61(suppl II) (2007) pp. ii46-ii53.

Ruiz Cantero, M. Teresa, Papí Gálvez, Natalia, Carbrera, Ruiz Virginia, Ruiz Martínez Ana, ÁlVAREz-DARdet DíAz Carlos. Los sistemas de género y/en la Encuesta Nacional de Salud. Gac Sanit 20 (2006) pp 427-34.

Ruiz, M. Teresa; VerbrugGe, Lois M. A two way view of gender bias in medicine. J Epidemiol Community Health. 51 (1997) pp. 106-9.

SEgura, Andreu. Zurriaga, O: RoHLFs, Isabel et al. diferencias por género en las características, manejo y letalidad-complicaciones en pacientes con infarto agudo de miocardio (IAM): Estudiolberica. Gac Sanit 16 (Suppl 1) (2002) pp 90.

Takkouche, Bahi, Regueira-Méndez, Carlos, Montes-Martínez, Agustín N. Risk of cancer among hairdressers and related workers: a meta-analysis. Int J Epidemiol 38 (2009) pp 1512-31.

Van der Wal, Jan F; Hoogeveen, Ank W.; Moons, André M.; Wouda, Paul. Investigation on the exposure of hairdressers to chemical agents. Environment International 23 (1997) pp 433-9.

WHITEHEAD, Margaret. The concepts and principles of equity and health. Copenhagen: WHO Regional Office for Europe; 1990. (Disponible en http://salud.ciee. flacso.org.ar/flacso/optativas/equity_and_health.pdf, consultado el 7/7/2011)

Feminismo/s 18, diciembre 2011, pp. 353-363 